$10 / 12 i 191750(1)$
SANDIA REPORT

SAND91-8500 UC-405

Unlimited Release

Printed August 1991

\title{
Massively Parallel Hypercube FFTs: CM-2 Implementation and Error Analysis of a Parallel Trigonometric Factor Generation Method
}

C. H. Tong and P. N. Swartztrauber

Prepared by

Sandia National Laboratories

Albuquerque. New Mexico 87185 and Livermore, California 94551

for the United States Department of Energy

under Contract DE-AC04-76DP00789 
Issued by Sandia National Laboratories, operated for the United States Department of Energy by Sandia Corporation.

NOTICE: This report was prepared as an account of work sponsored by an agency of the United States Government. Neither the United States Government nor any agency thereof, nor any of their employees, nor any of the contractors, subcontractors, or their employees, makes any war. ranty, express or implied, or assumes any legai liability or responsibility for the accuracy, completeness, or usefulness of any information, for the accuracy, completeness, or usefulness of any information,
apparatus, product, or process disclosed, or represents that its use would not infringe privately owned rights. Reference herein to any specific commercial product, process, or service by trade name, tradernark, manufacturer, or otherwise, does not necessarily constitute or imply its endorsement, recommendation, or favoring by the United States Government, any agency thereof or any of their contractors or subconractors. The views and opinions expressed herein do not necessarily state or reflect those of the United States Government, any agency thereof or any of their contractors or subcontractors.

This report has been reproduced from the best available copy.

Available to DOE and DOE contractors from:

Office of Scientific and Technical Information

P.O. Box 62

Oak Ridge TN 37831

Prices available from (615) 576-8401, FTS 626-8401.

Available to the public from:

National Technical information Service

U.S. Department of Commerce

5285 Port Royal Rd.

Springfield, VA 22161 


\author{
SA ND 91-8500 \\ Unlimited Release \\ Printed Augusî 1991
}

\title{
Massively Parallel Hypercube FFTs : CM-2 Implementation and Error Analysis of a Parallel Trigonometric Factor Generation Method
}

\author{
Charles Tong \\ Center for Computational Engineering, \\ Sandia National Laboratories, \\ Livermore, California 94551-0969 \\ Paul N. Swarztrauber \\ National Center for Atmospheric Research, \\ Boulder, Colorado 80307
}

July 31,1991

\begin{abstract}
On parallel computers, the way the data elements are mapped to the processors may have a large effect on the timing performance of a given algorithm. In our previous paper [16], we have examined a few mapping strategies for the ordered radix-2 DIF (decimation-in-frequency) Fast Fourier Transform. In particular, we have shown how reduction of communication can be achieved by combining the order and computational phases through the use of i-cycles [15]. A parallel method was also presented for computing the trigonometric factors which requires neither trigonometric function evaluation nor interprocessor communication. This paper first reviews some of the experimental results on the Connection Machine to demonstrate the importance of reducing communication in a parallel algorithm. The emphasis of this paper, however, is on analyzing the numerical stability of the proposed method for generating the trigonometric factors and showing how the error can be improved.
\end{abstract}

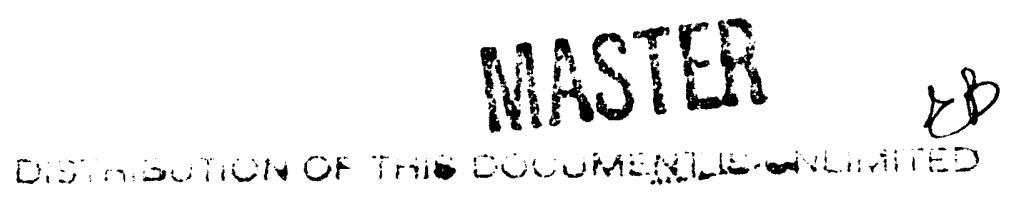




\section{Introduction}

The efficient implementation of the Fast Fourier Transform algorithm has been studied extensively on a wide variety of modern vector and parallel computers giving rise te its many variants. On vector computers, one main difference between the variants is the way the intermediate sequences are ordered to favor certain architectural characteristics. For example, intermediate orderings that give long vectors with unit stride are selected for most vector computers [14]. On hypercube multiprocessors, it is the mapping of initial and intermediate data elements to processors that dictates the amount of communication required [15] which in turn affects its overall performance.

In our previous paper [16] we have examined efficient implementation of ordered radix-2 DIF FFTs (for the rest of this paper, FFT will mean radix-2 DIF FFT unless otherwise indicated) on a massively parallel hypercube computers - the Connection Machine. Two mapping strategies were considered, namely, standard-map (or consecutive-map as used in [7]) and cyclic-map ([7] or scatter-map as used in [3]). Using the FFT with standard-map and i-cycles (defined later), a sequence with $N=2^{r}$ elements could be transformed with $r / 2+d+1$ parallel transmissions on a hypercube with $P=2^{d}$ processors if $d>r / 2[15]$. On the other hand, using the FFT with cyclic-map and $\mathrm{i}$-cycles, the same sequence can be computed with $2 d-r / 2$ parallel transmissions [16] (the term "parallel transmission" will also be defined later). Besides reducing the amount of communication, we have also shown that these transforms facilitate the parallel computation of the trigonometric factor without the need for trigonometric function evaluation nor interprocessor communication. This paper examines more closely on the parallel method for generating the trigonometric factors in terms of its numerical stability. In particular, we prove that the error growth of such a method is bounded above by $O\left(n^{1.5}\right)$ where $n$ is the length of the sequence to be transformed. We also show that this bound can be improved by using the idea of "restarting".

In order to retain the coherency of the complete FFT algorithm which includes the use of better mapping strategies, $\mathrm{i}$-cycles, and the new method for generating trigonometric factors, much of the contents covered in our previous paper [16] are included in this paper also. Thus, in section 2 , We first review the concept of $i$-cycle which is central to the efficient implementation of ordered hypercube FFT. We then describe two data sequence to processor mapping strategies - the standard-map and the cyclic-map, and study the communication requirements of these two mapping strategies together with the use of i-cycles. In section 3 , we first discuss different ways of computing the trigonometric factors and then present a parallel method for the direct computation of the trigonometric factors. Next we show that this method is particularly suited to a hypercube implementation using $\mathrm{i}$-cycles. The performance results of these FFTs are presented in section 4. Finally, an error analysis of the method for trigonometric factor generation is presented in section 5 .

\section{Hypercube FFT Algorithms}

\section{$2.1 \quad$ Introduction}

We consider the implementation of ordered FFTs on hypercube multiprocessors. It is assumed that the number of physical processors is $P=2^{d}$ where $d$ is the dimension of the hypercube. Each processor has its own local memory (also called distributed-memory system). It is also assumed that the number of elements to be transformed is $N=2^{r}$ and that $N / P$ is a small constant (massively parallel version of the original hypercube FFT [15]). Moreover, if $N / P>2$ (number of elements is more than twice the number of physical processors), the elements are mapped to virtual processors each of which contain exactly two elements (after the CM model). 
It is known that interprocessor communication consumes a substantial amount of time and hence its minimization is of primary concern. Communication between virtual processors located in the same physical processor does not contribute to interprocessor communication. Throughout the text we will use the following notation.

If $x_{n}$ has $N=2^{r}$ elements then it can be mapped in to the multidimensional array $x\left(i_{r-1}, \cdots, i_{0}\right)$ where $i_{r-1} i_{r-2}, \cdots, i_{0}$ is the binary form of $n$. The FFT can then be loosely described as a sequence of $2^{r-1}$ transforms of length two in each of $r$ dimensions. An example for the case $N=16$ is given in Table 1.

Table 1: Intermediate Orderings for Cooley-Tukey FFT, $N=16$ Binary Notation

$$
\begin{array}{|c|}
\hline x\left(i_{3} i_{2} i_{1} i_{0}\right) \\
X^{(1)}\left(k_{3} i_{2} i_{1} i_{0}\right) \\
X^{(2)}\left(k_{3} k_{2} i_{1} i_{0}\right) \\
X^{(3)}\left(k_{3} k_{2} k_{1} i_{0}\right) \\
X^{(4)}\left(k_{3} k_{2} k_{1} k_{0}\right) \\
X^{(4 a)}\left(k_{0} k_{1} k_{2} k_{3}\right) \\
\hline
\end{array}
$$

The original sequence is given as the first entry in Tabie 1 . The transform in the dimension $i_{3}$ is designated by replacing $i_{3}$ by $k_{3}$ in the second entry. Subsequent multiple 1-D transforms correspond to subsequent entries in Table 1. The FFT requires the multiple 1-D transforms to be computed in the order of decreasing indices, i.e., $i_{3}, i_{2}, i_{1}$, and $i_{0}$. The last entry corresponds to the bit-reversal that is necessary to order the FFT. Between each of the multiple 1-D transforms the sequence $x_{n}$ is multiplied by certain roots of unity. For example, $X^{(1)}\left(k_{3}, i_{2}, i_{1}, i_{0}\right)$ is computed from

$$
\begin{gathered}
X^{(1)}\left(0, i_{2}, i_{1}, i_{0}\right)=x\left(0, i_{2}, i_{1}, i_{0}\right)+x\left(1, i_{2}, i_{1}, i_{0}\right) \\
X^{(1)}\left(1, i_{2}, i_{1}, i_{0}\right)=\omega^{i_{2} i_{1} i_{0}}\left[x\left(0, i_{2}, i_{1}, i_{0}\right)-x\left(1, i_{2}, i_{1}, i_{0}\right)\right]
\end{gathered}
$$

where $\omega=e^{-i \pi / 4}$.

The last two entries in Table 1 correspond to a reordering in which the element in position $k_{3} k_{2} k_{1} k_{0}$ binary is moved to position $k_{0} k_{1} k_{2} k_{3}$.

\subsection{Index Digit Permutation and i-cycle}

The last entry in Table 1 is an example of an index-digit permutation [4], called a bit-reversal. Other examples include the perfect shuffle and matrix transpositions. The time required for communication is known to contribute substantially to the overall computing time. It is also known to depend significantly on how the sequence $x_{n}$ is mapped to the processors. We will begin with perhaps the most common mapping in which the first $N / P$ elements are mapped to the first processor, the second $N / P$ elements are mapped to the second processor and so forth.

Definition $1 A$ "standard" (or consecutive as in [7]) sequence to processor map (or standardmap) $x\left(i_{r-1} \cdots i_{r-d} \mid i_{r-d-1} \cdots i_{0}\right)$ is one in which the element $x_{n}$ with $n=i_{r-1} i_{r-2} \cdots i_{0}$ (binary) has address $i_{r-d-1} \cdots i_{0}$ in processor number $i_{r-1} i_{r-2} \cdots i_{r-d}[15]$.

Both a processor number and address are required to identify a particular element in the sequence. The partition / is introduced for expository purposes to separate the address on the right from the processor number on the left. For example if $r=4$ and $d=2$ then the element 
$x_{n}$ with $n=i_{3} i_{2} i_{1} i_{0}$ has address $i_{1} i_{0}$ and is located in processor number $i_{3} i_{2}$ and the mapping is designated by $x\left(i_{3} i_{2} \mid i_{1} i_{0}\right)$.

Definition 2 An (binary) index-digit permuted sequence to processor map is one in which the indices $i_{j}$ are permuted. That is, the element $x_{n}$ with $n=i_{r-1} i_{r-2} \cdots i_{0}$ (binary) has address $i_{m(r-d-1)} i_{m(r-d-2)} \cdots i_{m(0)}$ in processor number $i_{m(r-1)} i_{m(r-2)} \cdots i_{m(r-d)}$ where $m(j)$ is an arbitrary permutation of the integers $0, \cdots, r-1$ [15].

From the last two entries in Table 1 it is evident that a method will be needed for performing the index digit permutation on th: hypercube. To that end we introduce a specific class of communication tasks.

Definition 3 An (binary) i-cycle is an (binary) index-digit permutation of $x_{n}$ in which the most significant digit of the address (called the pivot) is exchanged with any other digit, either in the address or the processor number [15].

For example, if a standard sequence to processor map is used for $x_{n}$, an i-cycle is a reordering that exchanges the digit in position $r-d-1$ with any other digit. Two i-cycle examples are given in Table 2.

Table 2: Sample i-cycles for the case $d=2$ and $r=4$

$$
\begin{array}{|l|l|}
\hline X\left(i_{3} i_{2}\right. & \left.i_{1} i_{0}\right) \\
X\left(i_{3} i_{2}\right. & \left.i_{0} i_{1}\right) \\
X\left(i_{0} i_{2}\right. & \left.i_{3} i_{1}\right) \\
\hline
\end{array}
$$

The second entry in Table 2 is obtained from the first by an i-cycle that exchanges the first and second (pivot) digits. The third entry is obtained from the second by an i-cycle that exchanges the second and fourth digits. For $N=16$ and $P=4$ the data exchanges for two sample i-cycles are given in Table 3 below. The first two columns as well as the last two columns show the indices of the elements residing in the corresponding processors (the processor numbers are indicated in the third column) before and after an i-cycle has been performed. The i-cycles consist of parallel exchanges of packets with $N /(2 P)$ elements and for the two examples in Table 3 the packet size is $\frac{16}{2 \times 4}=2$. For example, the first two columns of Table 3 show that The third and fourth elements in processor 0 (i.e. 0010 and 0011 ) are exchanged with the first two elements in processor 1 (i.e. 0100 and 0101 ) while the third and fourth elements in processor 2 (i.e. 1010 and 1011) are exchanged with the first two elements in processor 3 (i.e. 1100 and 1101).

The i-cycle has three properties that make it useful for the development of parallel communication algorithms on the hypercube.

I-cycle property $\mathbf{A}$ : An i-cycle may or may not require interprocessor communication, depending on whether or not the digit is in the processor number. For example, the first i-cycle in Table 2 does not require interprocessor communication because the processor number is unchanged. However the second $\mathrm{i}$-cycle does require interprocessor communication because the processor number is changed. When interprocessor communication is required it is between processors that are directly connected because the processor numbers differ in only one bit. Through this discussion we are assuming that the sequence to processor map is an index-digit permuted map. A direct connection would not be established if the underlying map was (for example) a binary Gray code. 
Table 3: Sample i-cycle communication paths for $N=16$ and $P=4$

\begin{tabular}{|c|c|c|c|c|}
\hline \multicolumn{2}{|c|}{$X\left(i_{3} i_{2}\right.$} & $\left.i_{1} i_{0}\right)$ & & \multicolumn{2}{|c|}{$X\left(i_{3} i_{2}\right.$} & $\left.i_{1} i_{0}\right)$ \\
$X\left(i_{3} i_{1}\right.$ & $\left.i_{2} i_{0}\right)$ & & \multicolumn{2}{|c|}{$X\left(i_{1} i_{2}\right.$} \\
$\left.i_{3} i_{0}\right)$ \\
\hline$i_{3} i_{2} i_{1} i_{0}$ & $i_{3} i_{1} i_{2} i_{0}$ & $p$ & $i_{3} i_{2} i_{1} i_{0}$ & $i_{1} i_{2} i_{3} i_{0}$ \\
\hline 0000 & 0000 & 0 & 0000 & 0000 \\
0001 & 0001 & 0 & 0001 & 0001 \\
0010 & 0100 & 0 & 0010 & 1000 \\
0011 & 0101 & 0 & 0011 & 1001 \\
0100 & 0010 & 1 & 0100 & 0100 \\
0101 & 0011 & 1 & 0101 & 0101 \\
0110 & 0110 & 1 & 0110 & 1100 \\
0111 & 0111 & 1 & 0111 & 1101 \\
1000 & 1000 & 2 & 1000 & 0010 \\
1001 & 1001 & 2 & 1001 & 0011 \\
1010 & 1100 & 2 & 1010 & 1010 \\
1011 & 1101 & 2 & 1011 & 1011 \\
1100 & 1010 & 3 & 1100 & 0110 \\
1101 & 1011 & 3 & 1101 & 0111 \\
1110 & 1110 & 3 & 1110 & 1110 \\
1111 & 1111 & 3 & 1111 & 1111 \\
\hline
\end{tabular}

I-cycle property $B:$ It can be shown that at each stage of the FFT the packets transmitted between processors each contains $2^{r-d-1}=N /(2 P)$ elements and that $P / 2$ packets are exchanged in parallel.

I-cycle property $\mathbf{C}$ : Any index-digit permutation ca" be implemented as a sequence of $\mathrm{i}$ cycles. To see this, first decompose the permutation into disjoint cycles. Next decompose each cycle into $\mathrm{i}$-cycles by interchanging the first position with the pivot position and restore it following the completion of the cycle. For example, if the cycle is $(2,8,7,5)$ and the pivot is in position 3 , then this cycle is equivalent to the $\mathrm{i}$-cycles $(3,2)(3,8)(3,7)(3,5)(3,2)$ applied from left to right. Any index permutation can be implemented in no more than $1.5 d$ i-cycles [15].

\subsection{The Standard-order FFT}

Consider now the implementation of a "standard-order FFT" which is defined as one using standard-map and i-cycles. The $\mathrm{i}$-cycles are given in Table 4 for the case $r=8$ and $d=5$. The subscripts of the digits are increasing for a transform in standard order like the last entry in Table 1. The letter ' $a$ ' in the superscript indicates an ordering rather than computational step and an '*' following an entry indicates that a parallel transmission was necessary for that step. The sequence of $\mathrm{i}$-cycles is selected based on the theory presented in [15] where it is shown that for even $r>d / 2$ a total of $r / 2+d+1=10$ parallel transmissions are required. 
Table 4: Intermediate Orderings for a standard order FFT for $N=256$ and $P=32$

\begin{tabular}{|c|}
\hline$x\left(i_{7} i_{6} i_{5} i_{4} i_{3} \mid i_{2} i_{1} i_{0}\right)$ \\
\hline$X^{(1)}\left(i_{2} i_{6} i_{5} i_{4} i_{3} \mid k_{7} i_{1} i_{0}\right)^{*}$ \\
$X^{(2)}\left(i_{2} k_{7} i_{5} i_{4} i_{3} \mid k_{6} i_{1} i_{0}\right)^{*}$ \\
$X^{(3)}\left(i_{2} k_{7} k_{6} i_{4} i_{3} \mid k_{5} i_{1} i_{0}\right)^{*}$ \\
$X^{(4)}\left(i_{2} k_{7} k_{6} k_{5} i_{3} \mid k_{4} i_{1} i_{0}\right)^{*}$ \\
$X^{(5)}\left(i_{2} k_{7} k_{6} k_{5} k_{4} \mid k_{3} i_{1} i_{0}\right)^{*}$ \\
$X^{(5 a)}\left(i_{2} k_{7} k_{6} k_{3} k_{4} \mid k_{5} i_{1} i_{0}\right)^{*}$ \\
$X^{(6)}\left(k_{5} k_{7} k_{5} k_{3} k_{4} \mid k_{2} i_{1} i_{0}\right)^{*}$ \\
$X^{(6 a)}\left(k_{5} k_{7} k_{2} k_{3} k_{4} \mid k_{6} i_{1} i_{0}\right)^{*}$ \\
$X^{(7)}\left(k_{5} k_{7} k_{2} k_{3} k_{4} \mid k_{1} k_{6} i_{0}\right)$ \\
$X^{(7 a)}\left(k_{5} k_{1} k_{2} k_{3} k_{4} \mid k_{7} k_{6} i_{0}\right)^{*}$ \\
$X^{(8)}\left(k_{5} k_{1} k_{2} k_{3} k_{4} \mid k_{0} k_{6} k_{7}\right)$ \\
$X^{(8 a)}\left(k_{0} k_{1} k_{2} k_{3} k_{4} \mid k_{5} k_{6} k_{7}\right)^{*}$ \\
\hline
\end{tabular}

\subsection{The Cyclic-order FFT}

The mapping of a sequence on to the processors is known to significantly influence the time that is required for communication and hence mappings that reduce communication are of considerable interest. The difficulty with selecting a map that minimizes communication for a particular algorithm is that it niay not be optimum for a different part of the overall computation. However without knowledge of the other algorithms, and their optimal maps, it is not unreasonable to permit orderings other than the standard order. If order is not a consideration then it is known that the FFT can be performed with $d$ parallel transmissions. However it is likely that the other parts of the overall computation will expect the order of the transform and the input sequence to be the same, particularly if utilities and subroutines are used. Therefore we define an ordered transform as any transform in which the order of the sequence and its transform are the same.

In this section we will consider a variant of the parallel FFT presented above in which another mapping strategy and i-cycles are used. Communication is reduced and, as mentioned in the introduction, it is just as simple to select this map as the standard-map on the CM using geometry and priorities.

Definition $4 A$ "cyclic" sequence to processor map (or cyclic-map) $x\left(i_{d-1} \cdots i_{0} \mid i_{r-1} \cdots i_{d}\right)$ is one in which the element $x_{n}$ with $n=i_{r-1} i_{r-2} \cdots i_{0}$ (binary) has address $i_{r-1} i_{r-2} \cdots i_{d}$ in processor number $i_{d-1} i_{1} \cdots i_{0}[7]$.

A "cyclic-order FFT" is an ordered FFT using cyclic mapping and i-cycles. It requires fewer parallel transmissions than a standard-order FFT. An example is given in Table 5 for $N=256$ and $P=32$. As before, the locations that correspond to the digits on the right of the partition ' $"$ ' reside in the same physical processor. The digits on the left of the partition correspond to the processor number. An entry that ends with $a^{~}{ }^{*}$ ' indicates a parallel transmission and the lines with superscripts that end with a ' $a$ ' involve only communication.

The communication complexity for a cyclic-order FFT on parallel hypercube is given in the following lemma.

Lemma 1 A cyclic-order FFT of length $N=2^{r}$ can be implemented on a hypercube of dimension 
Table 5: Intermediate Orderings for a cyclic-order FFT with $N=256$ and $P=32$

\begin{tabular}{|c|}
\hline$x\left(i_{4} i_{3} i_{2} i_{1} i_{0} \mid i_{7} i_{6} i_{5}\right)$ \\
$X^{(1)}\left(i_{4} i_{3} i_{2} i_{1} i_{0} \mid k_{7} i_{6} i_{5}\right)$ \\
$X^{(2)}\left(i_{4} i_{3} i_{2} i_{1} i_{0} \mid k_{6} k_{7} i_{5}\right)$ \\
$X^{(3)}\left(i_{4} i_{3} i_{2} i_{1} i_{0} \mid k_{5} k_{7} k_{6}\right)$ \\
$X^{(4)}\left(k_{5} i_{3} i_{2} i_{1} i_{0} \mid k_{4} k_{7} k_{6}\right)^{*}$ \\
$X^{(5)}\left(k_{5} k_{4} i_{2} i_{1} i_{0} \mid k_{3} k_{7} k_{6}\right)^{*}$ \\
$X^{(5 a)}\left(k_{3} k_{4} i_{2} i_{1} i_{0} \mid k_{5} k_{7} k_{6}\right)^{*}$ \\
$X^{(6)}\left(k_{3} k_{4} k_{5} i_{1} i_{0} \mid k_{2} k_{7} k_{6}\right)^{*}$ \\
$X^{(6 a)}\left(k_{3} k_{4} k_{5} i_{1} i_{0} \mid k_{6} k_{7} k_{2}\right)$ \\
$X^{(7)}\left(k_{3} k_{4} k_{5} k_{6} i_{0} \mid k_{1} k_{7} k_{2}\right)^{*}$ \\
$X^{(7 a)}\left(k_{3} k_{4} k_{5} k_{6} i_{0} \mid k_{7} k_{1} k_{2}\right)$ \\
$X^{(8)}\left(k_{3} k_{4} k_{5} k_{6} k_{7} \mid k_{0} k_{1} k_{2}\right)^{*}$ \\
\hline
\end{tabular}

$d$ (where $d>r / 2$ ) with $2 d-r / 2$ parallel transmissions if $r$ is even and $2 d-(r-1) / 2$ parallel transmissions if $r$ is odd.

Proof : See [16].

The cyclic-order transform in Table 5 requires six parallel transmissions compared with ten for the standard-order FFT in Table 4. In general the cyclic-order FFT requires anywhere from $d$ to $1.5 d$ parallel transmissions and the standard-order FFT requires anywhere from $1.5 d$ to $2 d$ parallel transmissions. More specifically, for $d>r / 2$, the cyclic-order FFT requires $2 d-r / 2$ transmissions compared to $d+r / 2+1$ for the standard-order FFT. Therefore the cyclic-order FFT requires $r-d+1$ fewer parallel transmissions than the standard-order FFT. For the finest grain computations with $d=r-1$ they differ by only two parallel transmissions. Nevertheless this difference will likely be noticeable because the total communication time is proportional to $O(\log N)$ which is also a small integer.

The FFT is often a part of a larger computation that is posed on a grid so it is reasonable to ask about the compatibility of the Binary Reflected Gray code ordering and cyclic-ordering. In both the standard-order and the cy-lic-order transform the processors can be mapped so that nearest neighbors are at a distance of one, but at the expense of the $i$-cycles being conducted at a distance of two.

The cyclic-order hypercube FFT algorithm, written in pseudocode (similar to CM FORTRAN) is included $\mathrm{i}$. the following. The variable declaration and initialization have not been included.

C Cyclic-order hypercube FFT

$\mathrm{C} \mathrm{k}: \log 2(\mathrm{n})-1$

Subroutine FFT

do $\mathrm{i}=\mathrm{k}, 0,-1$

if $(i \neq k)$ call icycle(i) /* I-cycle */

call calculate_factor $/ *$ Calculate trigonometric factor $* /$

temp $=$ data $1+$ data $2 / *$ Compute new data points * $/$

data $2=($ data $1-$ data 2$) *$ twiddle

data $1=$ temp 


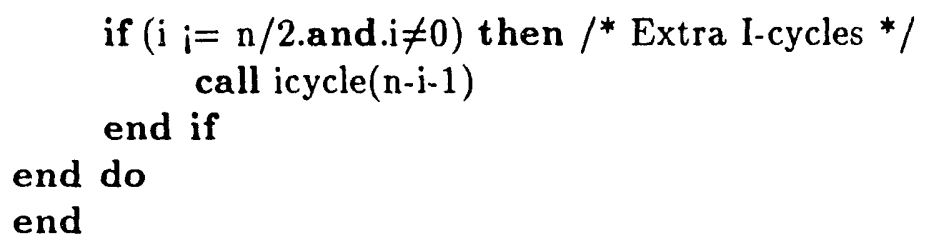

\section{Computing the Trigonometric Factors}

There are a few alternative methods for computing the trigonometric factors depending on the ayailable memory, I/O bandwidth, and processing capabilities [9].

Recursion All of the trigonometric factors at each stage are generated by recursion. This scheme requires only $O(1)$ storage and is popular on a uniprocessor or vector processors. However, the computation is highly sequential and not suitable for multiprocessors.

Table look-up The trigonometric factors are precomputed and stored in each processor. This scheme has an advantage for many FFTs since the trigonometric factors would be available for use without recalculation. However, this scheme also requires a large amount of storage $(O(\log N)$ per processor) for naive implementation. [8], for example, shows how to improve this memory utilization to $O(N+P \log P)$.

Direct calculation The trigonometric factors can be computed directly from the equation $W^{-k}=\cos (2 k \pi / N)-i \sin (2 k \pi k / N)$. However, the calculation of the trigonometric functions on each stage is very time consuming. Particularly since the FFT itself requires only a few operations.

Permutation Initially, the trigonometric factors are distributed among the processors according to the calculations required in the first stage. In the subsequent stages, half of the trigonometric factors are permuted each to two other processors. This scheme may be inefficient on parallel machine such as the CM where communication is expensive.

None of these methods are completely satisfactory on massively parallel computers if memory is limited and communication is expensive. However, by performing a few additional operations at each stage, the trigonometric factors can be computed in parallel without any communication.

Consider the following example of a 16-point FFT (unordered transform) and suppose that element $\mathrm{i}$ is mapped to processor $\mathrm{i}$, then the trigononietric factors needed at each stage of the algorithm are as in Table 6. The entries in each column correspond to $k$ in the trigonometric factor $W^{-k}$. Entries with the form $(k)$ refer to the exponent of a trigonometric factor that is not used at the current stage but is needed to compute the factors at a subsequent stage of the FFT using the proposed scheme.

It can be seen that the integers in each column are twice $(\bmod N / 2)$ the integers in the previous column and henc: the trigonometric factors can be computed from the identities.

$$
\begin{gathered}
\cos 2 \theta=\cos ^{2} \theta-\sin ^{2} \theta \quad, \text { and } \\
\sin 2 \theta=2 \cos \theta \sin \theta .
\end{gathered}
$$

Thus, we can calculate the trigonometric factors for the current stage from the previous stage by four multiplications and one addition (or three multiplications and two additions). This method can also be used to generate the table for the table look-up scheme. One drawback of this 
Table 6: Trigonometric Coefficients for a 16-point unordered FFT

\begin{tabular}{|c|c|c|c|c|}
\hline Processor & \multicolumn{4}{|c|}{ Value of $k$ in $W^{-k}$} \\
\hline Processor Number (binary) & Stage 1 & Stage 2 & Stage 3 & Stage 4 \\
\hline 0000 & $(0)$ & $(0)$ & $(0)$ & $(0)$ \\
0001 & $(1)$ & $(2)$ & $(4)$ & 0 \\
0010 & $(2)$ & $(4)$ & 0 & $(0)$ \\
0011 & $(3)$ & $(6)$ & 4 & 0 \\
0100 & $(4)$ & 0 & $(0)$ & $(0)$ \\
0101 & $(5)$ & 2 & $(4)$ & 0 \\
0110 & $(6)$ & 4 & 0 & $(0)$ \\
0111 & $(7)$ & 6 & 4 & 0 \\
1000 & 0 & $(0)$ & $(0)$ & $(0)$ \\
1001 & 1 & $(2)$ & $(4)$ & 0 \\
1010 & 2 & $(4)$ & 0 & $(0)$ \\
1011 & 3 & $(6)$ & 4 & 0 \\
1100 & 4 & 0 & $(0)$ & $(0)$ \\
1101 & 5 & 2 & $(4)$ & 0 \\
1110 & 6 & 4 & 0 & $(0)$ \\
1111 & 7 & 6 & 4 & 0 \\
\hline
\end{tabular}

scheme for the unordered transform is the need to generate extra factors which are not used (the ones in parentheses in Table 6 but are needed to generate subsequent factors. This drawback, as we shall see below, does not occur for the ordered (both cyclic-order and standard-order) FFTs presented in section 2. Table 7 contains the exponents for the cyclic-order transform with $N=16$ and $P=8\left(2\right.$ points per processor or $\left(0, i_{2}, i_{1}, i_{0}\right)$ and $\left(1, i_{2}, i_{1}, i_{0}\right)$ reside in the same processor initially).

Table 7: Trigonometric factors for a 16-point cyclic-order FFT

\begin{tabular}{|c|c|c|c|c|}
\hline Processor & \multicolumn{4}{|c|}{ Value of $k$ in $W^{-k}$} \\
\hline Processor Number (binary) & Stage 1 & Stage 2 & Stage 3 & Stage 4 \\
\hline 000 & 0 & 0 & 0 & 0 \\
001 & 1 & 2 & 4 & 0 \\
010 & 2 & 4 & 0 & 0 \\
011 & 3 & 6 & 4 & 0 \\
100 & 4 & 0 & 0 & 0 \\
101 & 5 & 2 & 4 & 0 \\
110 & 6 & 4 & 0 & 0 \\
111 & 7 & 6 & 4 & 0 \\
\hline
\end{tabular}

Fewer computations are required because every trigonometric factor is used and therefore a factor of two is saved compared to the unordered FFT. In general, this method of computing trigonometric factors can be used if the order of the not-yet-transformed bits $\left(i_{j}\right)$ is preserved. The characteristics of the methods for computing the trigonometric factors are summarized ir: 
Table 8.

Table 8: Characteristics of Different Methods for Computing Trigonometric Factors

\begin{tabular}{|c|c|c|c|c|}
\hline Method & storage & computation & comm. & comment \\
\hline recursion & $O(1)$ & $O(N \log N)$ & 0 & very sequential \\
\hline table look up & $O(N+P \log P)$ & $O(\log N)$ & 0 & reuseability \\
\hline permutation & $O(N)$ & $O(1)$ & $O(\log N)$ & - \\
\hline direct calculation & $O(N)$ & $O(\log N)$ & 0 & use sin and cos \\
\hline new method & $O(N)$ & $O(\log N)$ & 0 & no sin and cos \\
\hline
\end{tabular}

\section{Performance of the Parallel Hypercube FFTs on the CM-2}

In this section we consider the performance of three ordered FFTs on a hypercube whose logical and physical processor numbers are the same :

1. the standard-order FFT,

2. the cyclic-order FFT,

3. an ordered FFT by Hertz [6] which have separate bit-reversal and butterfly phases.

Using CM FORTRAN/PARIS it is possible to equate logical and physical processor numbers. That is, any reference to processor $i_{d-1} \ldots i_{0}$ is a reference to a processor with the same binary representation in the hypercube (as opposed to, for example, the binary reflected Gray code mapping). A significant improvement is obtained because the key communication task (i-cycle) is conducted at a physical distance of at most one using news communication for all $\mathrm{i}$-cycles. The programs were written in CM FORTRAN/PARIS and run on a CM-2. The times for different size FFTs are listed in Table 9 and the corresponding MFLOPS counts are listed in Table 10.

The MFLOPS for (3) is calculated using the same formula as (1) and (2). In reality, method (3) requires more than 7.5 operation per point and thus the MFLOPS count should be higher due to the need for computing the sine and cosine functions at each stage of the FFT.

These results demonstrate the attributes of cyclic-map, i-cycles, and the new parallel method of computing the trigonometric factors. From Table 10, we estimate a performance of about 0.9 GFLOPS for a $16 \mathrm{M}$-point FFT on a full $64 \mathrm{k}$ CM-2.

\section{Error Analysis of the New Method for Computing Trigono- metric Factors}

In this section we consider the numerical accuracy of our new method for generating the trigonometric factors. Both analytical and numerical results will be presented. We shall begin with a few definitions.

Definition 5 [5] If a number $x$ is represented by the nearest floating point number $(f l(x))$ with precision $t$ (number of digits for the mantissa) and machine base $b$, then

$$
f l(x)=x(1+\epsilon), \quad|\epsilon| \leq \mathbf{u}
$$


Table 9: Computing time in seconds for three ordered FFTs

\begin{tabular}{|c||c|c|c|c|}
\hline size FFT & machine size & FFT (1) & FFT (2) & FFT (3) \\
\hline \hline 131072 & $8 \mathrm{k}$ & 0.22 & 0.16 & - \\
\hline 262144 & $8 \mathrm{k}$ & 0.45 & 0.32 & - \\
\hline 524288 & $8 \mathrm{k}$ & 0.94 & 0.67 & - \\
\hline 1048576 & $8 \mathrm{k}$ & 1.92 & 1.39 & - \\
\hline 2097152 & $8 \mathrm{k}$ & 3.95 & 2.89 & - \\
\hline 262144 & $16 . \mathrm{k}$ & 0.23 & 0.17 & 0.688 \\
\hline 524288 & $16 \mathrm{k}$ & 0.49 & 0.36 & 1.40 \\
\hline 1048576 & $16 \mathrm{k}$ & 1.01 & 0.72 & 2.95 \\
\hline 2097152 & $16 \mathrm{k}$ & 2.07 & 1.50 & 6.10 \\
\hline 4194304 & $16 \mathrm{k}$ & 4.23 & 3.07 & 12.68 \\
\hline 524288 & $32 \mathrm{k}$ & 0.25 & 0.19 & - \\
\hline 1048576 & $32 \mathrm{k}$ & 0.52 & 0.39 & - \\
\hline 2097152 & $32 \mathrm{k}$ & 1.09 & 0.80 & - \\
\hline 4194304 & $32 \mathrm{k}$ & 2.22 & 1.59 & - \\
\hline 8388608 & $32 \mathrm{k}$ & 4.55 & 3.29 & - \\
\hline \hline \multicolumn{5}{|c}{ FFT (1) standard order FFT. } \\
\hline \multicolumn{5}{|c}{ FFT (2) cyclic-order FFT. } \\
\hline
\end{tabular}

Table 10: MFLOPS for three ordered FFTs

\begin{tabular}{|c|c|c|c|c|}
\hline size FFT & machine size & FFT (1) & FFT (2) & FFT (3) \\
\hline 131072 & $\overline{8 k}$ & 76 & 104 & - \\
\hline 262144 & $8 \mathrm{k}$ & 79 & 111 & - \\
\hline 524288 & $8 \mathrm{k}$ & 79 & 112 & - \\
\hline 1048576 & $8 \mathrm{k}$ & 82 & 113 & - \\
\hline 2097152 & $8 \mathrm{k}$ & 84 & 114 & - \\
\hline 262144 & $16 \mathrm{k}$ & 154 & 208 & 51 \\
\hline 524288 & $16 \mathrm{k}$ & 152 & 208 & 53 \\
\hline 1048576 & $16 \mathrm{k}$ & 156 & 218 & 53 \\
\hline 2097152 & $16 \mathrm{k}$ & 160 & 220 & 54 \\
\hline 4194304 & $16 \mathrm{k}$ & 164 & 225 & 55 \\
\hline 524288 & $32 \mathrm{k}$ & 299 & 393 & - \\
\hline 1048576 & $32 k$ & 302 & 403 & - \\
\hline 2097152 & $32 \mathrm{k}$ & 303 & 413 & - \\
\hline 4194304 & $32 \mathrm{k}$ & 318 & 435 & - \\
\hline 8388608 & $32 \mathrm{k}$ & 318 & 440 & - \\
\hline \multicolumn{5}{|c|}{$\begin{array}{l}\text { FFT (1) standard order FFT. } \\
\text { FFT (2) cyclic-order FFT. } \\
\text { FFT (3) P. Hertz FFT. [6] } \\
\text { ،.-s Data not available. }\end{array}$} \\
\hline
\end{tabular}


where $\mathbf{u}$ is the unit roundoff error defined by

$$
u=\frac{1}{2} b^{1-t}
$$

Definition 6 Let $c_{j}^{k}$ and $s_{j}^{k}$ be the exact required cosine and sine factors for element $j$ at stage $k$ of the FFT, then the corresponding computed $\hat{c}_{j}^{k}$ and $\hat{s}_{j}^{k}$ satisfies

$$
\begin{gathered}
\left|\hat{c}_{j}^{k}-c_{j}^{k}\right| \leq h(k) \mathbf{u}, \quad \text { and } \\
\left|\hat{s}_{j}^{k}-s_{j}^{k}\right| \leq h(k) \mathbf{u}
\end{gathered}
$$

where $h(k)$ is the growth rate of the error for our method.

Assumption : If $\hat{c}_{j}^{k}$ and $\hat{s}_{j}^{k}$ are computed through direct cosine and sine function calls to the library, then

$$
\begin{gathered}
\left|\hat{c}_{j}^{k}-c_{j}^{k}\right| \leq \mathbf{u}, \quad \text { and } \\
\left|\hat{s}_{j}^{k}-s_{j}^{k}\right| \leq \mathbf{u} .
\end{gathered}
$$

The algorithm for computing the trigonometric factors is as follow :

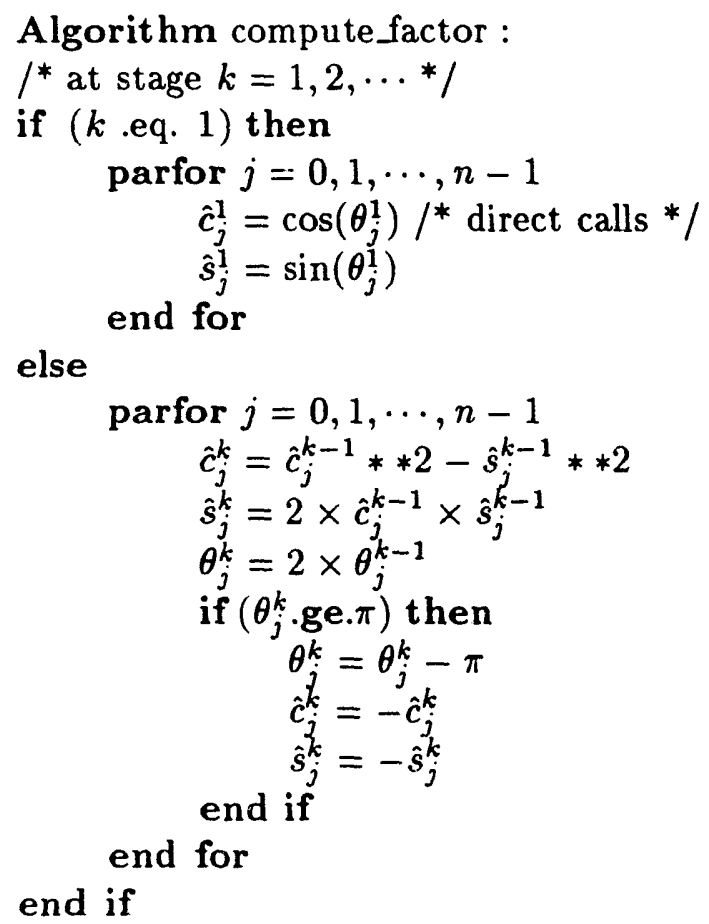

This method computes the trigonometric factors initially using direct calls to the cosine and sine library functions and by the above assumption,

$$
\begin{gathered}
\left|\hat{c}_{j}^{1}-c_{j}^{1}\right| \leq \mathbf{u}, \quad \text { and } \\
\left|\hat{s}_{j}^{1}-s_{j}^{1}\right| \leq \mathbf{u}, \quad \forall i .
\end{gathered}
$$

Lemma $2\left|\hat{c}_{j}^{k}-\left(\hat{c}_{j}^{k-1}\right)^{2}+\left(\hat{s}_{j}^{k-1}\right)^{2}\right| \leq 2 \mathbf{u}+\mathbf{u}^{2}$. 


\section{Proof :}

$$
\begin{aligned}
\hat{c}_{j}^{k}= & f l\left(f l\left(\hat{c}_{j}^{k-1} \hat{c}_{j}^{k-1}\right)-f l\left(\hat{s}_{j}^{k-1} \hat{s}_{j}^{k-1}\right)\right) \\
= & {\left[\left(\hat{c}_{j}^{k-1}\right)^{2}\left(1+\delta_{1}\right)-\left(\hat{s}_{j}^{k-1}\right)^{2}\left(1+\delta_{2}\right)\right]\left(1+\delta_{3}\right) } \\
= & \left(\hat{c}_{j}^{k-1}\right)^{2}-\left(\hat{s}_{j}^{k-1}\right)^{2}+\delta_{1}\left(\hat{c}_{j}^{k-1}\right)^{2}-\delta_{2}\left(\hat{s}_{j}^{k-1}\right)^{2}+\delta_{3}\left(\hat{c}_{j}^{k-1}\right)^{2}- \\
& \delta_{3}\left(\hat{s}_{j}^{k-1}\right)^{2}+\delta_{1} \delta_{3}\left(\hat{c}_{j}^{k-1}\right)^{2}-\delta_{2} \delta_{3}\left(\hat{s}_{j}^{k-1}\right)^{2}
\end{aligned}
$$

where $\left|\delta_{i}\right| \leq \mathbf{u}, i=1,2,3$ and which leads to the lemma by noting that

$$
\left|\hat{c}_{j}^{k}\right| \leq 1, \forall j, k . \quad \square
$$

Lemma $3\left|\hat{s}_{j}^{k}-2 \hat{c}_{j}^{k-1} \hat{s}_{j}^{k-1}\right| \leq 2 \mathbf{u}$.

Proof :

$$
\begin{aligned}
\hat{s}_{j}^{k} & =f l\left(2 \hat{c}_{j}^{k-1} \hat{s}_{j}^{k-1}\right) \\
& =2 \hat{c}_{j}^{k-1} \hat{s}_{j}^{k-1}\left(1+\delta_{4}\right)
\end{aligned}
$$

where $\left|\delta_{\mathbf{4}}\right| \leq \mathbf{u}$ and which again leads to the lemma.

\section{Lemma 4}

$$
\begin{gathered}
\left|\hat{c}_{j}^{k}-c_{j}^{k}\right| \leq 2 \sqrt{2} h(k-1) \mathbf{u}+2 \mathbf{u}+\mathbf{u}^{2}, \text { and } \\
\left|\hat{s}_{j}^{k}-s_{j}^{k}\right| \leq 2 \sqrt{2} h(k-1) \mathbf{u}+2 \mathbf{u}+\mathbf{u}^{2} .
\end{gathered}
$$

Proof :

$$
\begin{aligned}
\left|\hat{c}_{j}^{k}-c_{j}^{k}\right| & =\left|\hat{c}_{j}^{k}-\left(\hat{c}_{j}^{k-1}\right)^{2}+\left(\hat{s}_{j}^{k-1}\right)^{2}\right|+\left|\left(\hat{c}_{j}^{k-1}\right)^{2}-\left(\hat{s}_{j}^{k-1}\right)^{2}-\left(c_{j}^{k-1}\right)^{2}+\left(s_{j}^{k-1}\right)^{2}\right| \\
& \leq 2 \mathbf{u}+\mathbf{u}^{2}+\left|\left(\hat{c}_{j}^{k-1}\right)^{2}-\left(c_{j}^{k-1}\right)^{2}\right|+\left|\left(\hat{s}_{j}^{k-1}\right)^{2}-\left(s_{j}^{k-1}\right)^{2}\right| \\
& \leq 2 \mathbf{u}+\mathbf{u}^{2}+\left|\hat{c}_{j}^{k-1}+c_{j}^{k-1}\right|\left|\hat{c}_{j}^{k-1}-c_{j}^{k-1}\right|+\left|\hat{s}_{j}^{k-1}+s_{j}^{k-1}\right|\left|\hat{s}_{j}^{k-1}-s_{j}^{k-1}\right| \\
& \leq 2 \mathbf{u}+\mathbf{u}^{2}+\left(\left|\hat{c}_{j}^{k-1}\right|+\left|c_{j}^{k-1}\right|+\left|\hat{s}_{j}^{k-1}\right|+\left|s_{j}^{k-1}\right|\right) h(k-1) \mathbf{u} .
\end{aligned}
$$

Now since

$$
\begin{gathered}
\left(\hat{c}_{j}^{k-1}\right)^{2}+\left(\hat{s}_{j}^{k-1}\right)^{2}=1, \quad \text { and } \\
\left(c_{j}^{k-1}\right)^{2}+\left(s_{j}^{k-1}\right)^{2}=1,
\end{gathered}
$$

with a little trigonometry, it is straightforward to show that

$$
\left|\hat{c}_{j}^{k-1}\right|+\left|\hat{s}_{j}^{k-1}\right| \leq \sqrt{2} .
$$

Hence we have,

$$
\left|\hat{c}_{j}^{k}-c_{j}^{k}\right| \leq 2 \sqrt{2} h(k-1) \mathbf{u}+2 \mathbf{u}+\mathbf{u}^{2} .
$$

The same analysis can be carried uut for the computation of sinc function.

Finally, we have the following theorem : 
Theorem 1 If $\hat{c}_{j}^{k}$ and $\hat{s}_{j}^{k}$ are computed via Algorithm compute_factor, then

$$
\begin{gathered}
\left|\hat{c}_{j}^{k}-c_{j}^{k}\right| \leq h(k) \mathbf{u}+O\left(\mathbf{u}^{2}\right), \text { and } \\
\left|\hat{s}_{j}^{k}-s_{j}^{k}\right| \leq h(k) \mathbf{u}+O\left(\mathbf{u}^{2}\right),
\end{gathered}
$$

where $h(k)=\frac{1}{2 \sqrt{2}}(2 \sqrt{2})^{k}$.

Proof :

From the previous lemma, we have the following recurrence

$$
h(k)=2 \sqrt{2} h(k-1) .
$$

Solving this recurrence using initial condition $h(1)=1$ gives

$$
h(k)=\frac{1}{2 \sqrt{2}}(2 \sqrt{2})^{k} .
$$

Corollary 1 The maximum error given by Algorithm compute_factor is $\frac{1}{2 \sqrt{2}} n^{3 / 2}$.

This is obvious if we notice that $k$ only goes up to $\log _{2} n$.

An additional feature about this new method is that if the above error bound is not acceptable, we can restart (using direct calls), say, at the middle (stage $\frac{\log _{2} n}{2}$ ) of FFT and we have the following corollary.

Corollary 2 If the Algorithm compute-factor is restcirted stage $\frac{\log _{2} n}{2}$, then the maximum error incurred is $\frac{1}{2 \sqrt{2}} n^{3 / 4}$.

This corollary can be easily proved by noticing now that $k$ only goes up to $\frac{\log _{2} n}{2}$. If this bound is still not acceptable, we can have more restarts to achieve the desired accuracy.

It is given in [1] that the error for the Cooley Tukey FFT is of $O\left(h^{\text {method }} \log _{2} n\right)$ where $h^{\text {method }}$ is the error incurred in the trigonometric factor calculations. Hence, we have the final theorem.

Theorem 2 The error bound for the Cooley Tukey FFT using Algorithm compute_factor to com-

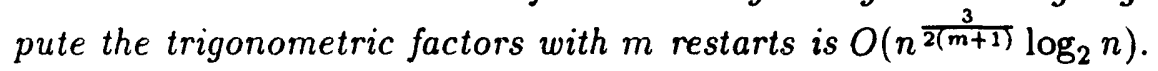

In the following we present numerical results using, single-precision arithmetic on the CM. A vector consisting of a discrete sine function in the real part and zero in the imaginary part is input to a FFT subroutine and the output is then input to an inverse FFT subroutine, both using the new method. An error vector is generated by taking the difference of the real parts of the original input and the final output. Table 11 shows the maximum norm and the root mean square of the error vector. The root mean square value is computed by taking the 2 -norm of the error vector and then divided it by the size of the input. Table 12 shows the same norms but the new method is used with one restart. We observe a large increase in accuracy with the use of just one restart. 
Table 11: Error magnitudes using the new method

\begin{tabular}{|c||c|c|}
\hline size FFT N & Maximum norm & 2 -norm/N \\
\hline 8192 & $6.43730 \mathrm{e}-4$ & $3.31699 \mathrm{e}-6$ \\
\hline 16384 & $1.44638 \mathrm{e}-3$ & $4.34077 \mathrm{e}-6$ \\
\hline 32768 & $3.05253 \mathrm{e}-3$ & $6.41415 \mathrm{e}-6$ \\
\hline 65536 & $6.69646 \mathrm{e}-3$ & $8.13486 \mathrm{e}-6$ \\
\hline 131072 & $1.65939 \mathrm{e}-2$ & $1.24870 \mathrm{e}-5$ \\
\hline 262144 & $3.11155 \mathrm{e}-2$ & $2.00082 \mathrm{e}-5$ \\
\hline 524288 & $6.67725 \mathrm{e}-2$ & $2.45073 \mathrm{e}-5$ \\
\hline
\end{tabular}

Table 12: Error magnitudes using the new method with 1 restart

\begin{tabular}{|c||c|c|}
\hline size FFT N & Maximum norm & 2 -norm/N \\
\hline 8192 & $2.44975 \mathrm{e}-5$ & $9.07233 \mathrm{e}-8$ \\
\hline 16384 & $3.37362 \mathrm{e}-5$ & $9.01880 \mathrm{e}-8$ \\
\hline 32768 & $4.61340 \mathrm{e}-5$ & $8.24786 \mathrm{e}-8$ \\
\hline 65536 & $8.73804 \mathrm{e}-5$ & $1.00031 \mathrm{e}-7$ \\
\hline 131072 & $1.00136 \mathrm{e}-4$ & $8.58351 \mathrm{e}-8$ \\
\hline 262144 & $1.65522 \mathrm{e}-4$ & $9.80134 \mathrm{e}-8$ \\
\hline 524288 & $2.08616 \mathrm{e}-4$ & $8.93721 \mathrm{e}-8$ \\
\hline
\end{tabular}

\section{Summary and Conclusion}

First, the experimental results in section 4 demonstrate that performance can be improved by combining the communication and computational phases [15] in an ordered transform. Although this result has been demonstrated on the $\mathrm{CM}$ it would also be true for any hypercube because communication time is a significant part of the overall computing time. Second, the cyclic-order FFT has performance that is superior to the standard-order FFT and is therefore recommended where applicable. In addition, a parallel algorithm for computing the trigonometric factors was presented that represents an attractive compromise between the communication, computation, and memory constraints that exist on the CM. The use of the i-cycle, cyclic-map, and the new parallel algorithm for computing the trigonometric factors have resulted in the development of a high performance ordered FFT for the CM.

\section{References}

[1] C. Chu, Fast Fourier Transforms on Hypercube Computers, Ph.D. Dissertation, Department of Computer Science, Cornell University, 1987.

[2] P. M. Flanders, A Unified Approach to a Class of Data Movements on an Array Processor. IEEE Trans. Computers, 31(9), pp.809-819, September 1982.

[3] G. C. Fox and S. W. Otto, Concurrent Computation and the theory of Complex systems, Technical Report CCCP-25., California Inst. of Technology, Pasadena, CA, March 1986. 
[4] D. Fraser, Array permutation by index-digit permutation, ACM, 22(1976), pp. 298-306.

[5] Golub, G. H., Van Loan, C. F., Matrix Computations, the Johns Hopkin University Press, 1983 , chapter 6.

[6] P. Hertz, An Algorithm for the Fast Fourier Transform On the Connection Machine, To appear in Computers in Physics.

[7] S. L. Johnsson, Communication Efficient Basic Linear Algebra Computations on Hypercube Architectures, J. Parallel Distributed Comput., 4(2):133-172, April 1987.

[8] S. L. Johnsson, C. T. Ho, M. Jacquemin and A. Ruttenberg, Computing Fast Fourier Transforms on Boolean Cubes and related networks, In Advanced Algorithms and Architectures for Signal Processing II, Vol. 826, pp. 223-231, 1987.

[9] R.A. Kamin III, and G.B. Adams III, Fast Fourier Transform Algorithm Design and Tradeoffs on the CM, Proceedings of the Conference on Scientific Applications of the Connection Machine, Editor : H. Simon, World Scientific Publishing Co., 1989.

[10] O.A. McBryan, Connection Machine Application Performance, CU-CS-434-89, Department of Computer Science, University of Colorado, April 1989.

[11] D. Nassimi and S. Sahni, An Optimal Routing Algorithm for Mesh-connected Parallel Computers, JACM, 27(1), pp.6-29, January 1980.

[12] D. Nassimi and S. Sahni, Optimal bpc Permutations on a Cube-connected SIMD Computer, IEEE Trans. Computers, C-31(4), pp.338-341, April, 1982.

[13] A.V. Oppenheim, and R.W. Schafer, Digital Signal Processing, Prentice Hall, 1975.

[14] P.N. Swarztrauber, FFT algorithms for vector computers, Parallel Computing, 1 (1984), pp. 45-63.

[15] P.N. Swarztrauber, Multiprocessor FFTs, Parallel Computing, 5 (1987), pp. 197-210.

[16] C. H. Tong and C. and P. Swarztrauber, Ordered Fast Fourier Transform on a Massively Parallel Hypercube Multiprocessor, to appear in Journal of Parallel and Distributed Computing. 


\section{UNUMITED RELEASE}

INITIAL DISTRIBUTION:

$\begin{aligned} & 1421 \text { J. N. Shadid } \\ & 1422 \text { R. S. Tuminaro } \\ & 8000 \text { J. C. Crawford } \\ & \text { Attn: E. E. Ives, } 8100 \\ & \text { P. L. Mattern, } 8300 \\ & \text { R. C. Wayne, } 8400 \\ & \text { P. E. Brewer, } 8500\end{aligned}$

8200

R. J. Detry,

8210 W. D. Wilson

8211 J. C. Meza

8211 C. L. Bisson

8211 M. E. Colvin

8211 D. B. McGarrah

8211 M. H. Pendley

8211 C. H. Tong (5)

$8211 \quad$ R. S Judson

$8300 A$ R. E. Cline

8535 Publications Division/Technical

Library Processes Division 3141

8535 Publications for OSTI (10)

3141 Technical Library Processes (3)

8524-2 Central Technical Files (3) 

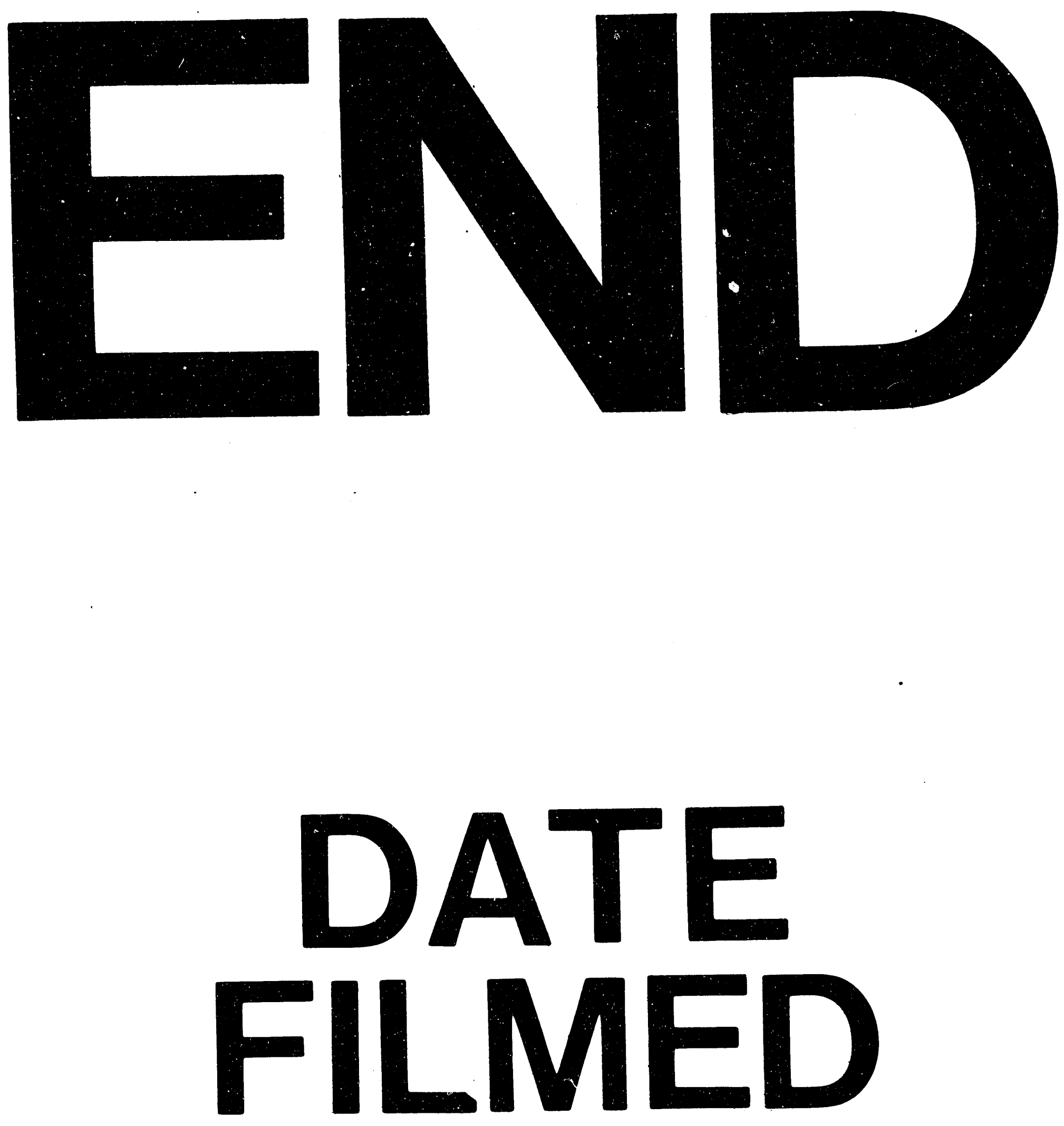

1

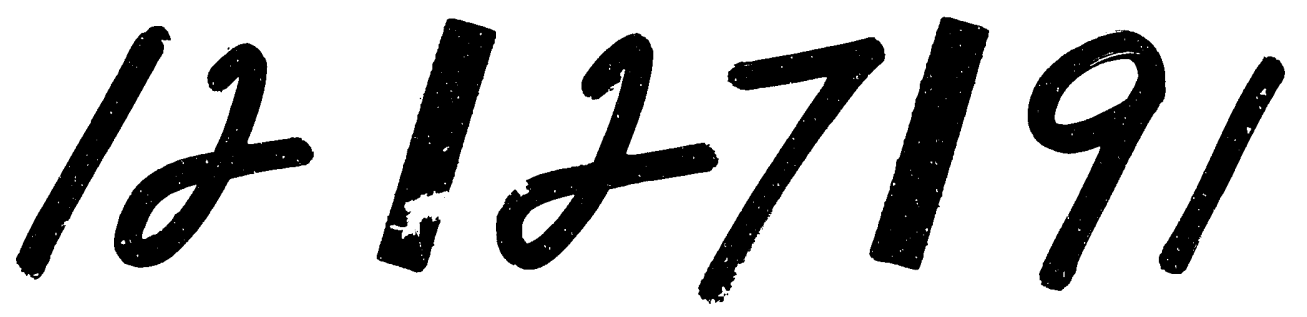


